\title{
AUTOMATIZACIÓN DEL PROCESO DE MACERADO DE LA MALTA PARA LA PRODUCCIÓN DE CERVEZA ARTESANAL
}

\author{
Automation of the maceration process of the malt \\ for the production of craft beer
}

EPISTEMUS

ISSN: 2007-8196 (electrónico)

ISSN: 2007-4530 (impresa)

Victor Hugo Benitez Baltazar 1 Cristian Omar Morales Rivas 1

Recibido: 14 de marzo de 2017

Aceptado: 30 de mayo de 2018

Autor de Correspondencia:

Dr. Victor Hugo Benitez Baltazar

Correo: vbnitez@industrial.uson.mx

\section{Resumen}

En este trabajo se presenta el diseño de un prototipo funcional que realizará la maceración de la malta de forma automatizada, el cual es un proceso muy importante en la producción de cerveza artesanal, dicho proyecto incluye el desarrollo de los códigos de programación de cada uno de los microcontroladores utilizados, la elaboración física del sistema, así como la comunicación y monitoreo a través del computador.

Palabras clave: Monitoreo, automatización, cerveza artesanal.

\section{Abstract}

This paper presents the design of a functional prototype that will perform the automation of the maceration process of the malt, which is a very important process in the production of craft beer, this project includes the development of the programming codes of each of the micro controllers Implemented, the physical elaboration of the system, as well as the communication and monitoring through the computer.

Keywords: Monitoring, automation, craft beer.

1 Universidad de Sonora / Departamento de Ingeniería Industrial / Ingeniería Mecatrónica / vbnitez@industrial.uson.mx 


\section{INTRODUCCIÓN}

Se denomina cerveza a una bebida alcohólica, no destilada, de sabor amargo que se fabrica con granos de cebada u otros cereales cuyo almidón, una vez modificado, es fermentado en agua, previamente aromatizado con lúpulo. De ella se conocen múltiples variantes con una amplia gama de matices debidos a las diferentes formas de elaboración y a los ingredientes utilizados, su aspecto puede ser cristalino o turbio, además su graduación alcohólica se encuentra generalmente entre los $3 \%$ y los $9 \%$ vol.

Toda cerveza se elabora con cuatro elementos básicos: cebada, agua, lúpulo y levadura. La diferencia principal entre la cerveza industrial y la artesanal se encuentra en las proporciones, en el tratamiento de la materia prima y en el proceso de elaboración. Las cervezas artesanales no utilizan ningún aditivo artificial, el proceso de elaboración es manual desde el molido de las maltas hasta el embotellamiento. La cerveza artesanal tiene su propio maestro cervecero y su propio tiempo para la elaboración. El maestro cervecero se informa acerca de los ingredientes y en cómo ir creando cada formula, es más tiempo y dedicación, por eso se puede encontrar una gran variedad de sabores o estilos en cada marca cervecera, y esto es lo que la diferencia de los demás productos del mercado. Es por esta razón que la cerveza artesanal tiene un costo más elevado que la comercial. En esta proyecto se diseñó un sistema automático de macerado, el cual es una de las fases del proceso de producción de la cerveza y sirve para tener un mayor control del proceso de producción, claro estás, sin dejar de lado que es un proceso artesanal y en esto se encuentra el valor agregado del producto.

\section{PRELIMINARES}

El proceso de maceración es un proceso de extracción entre materias de diferentes estados físicos de sólidolíquido, en el cual los compuestos químicos de interés se encuentran en el material sólido; ya que estos poseen solubilidad, se usa un líquido que permita su extracción. En el proceso de macerado se encuentran diferentes

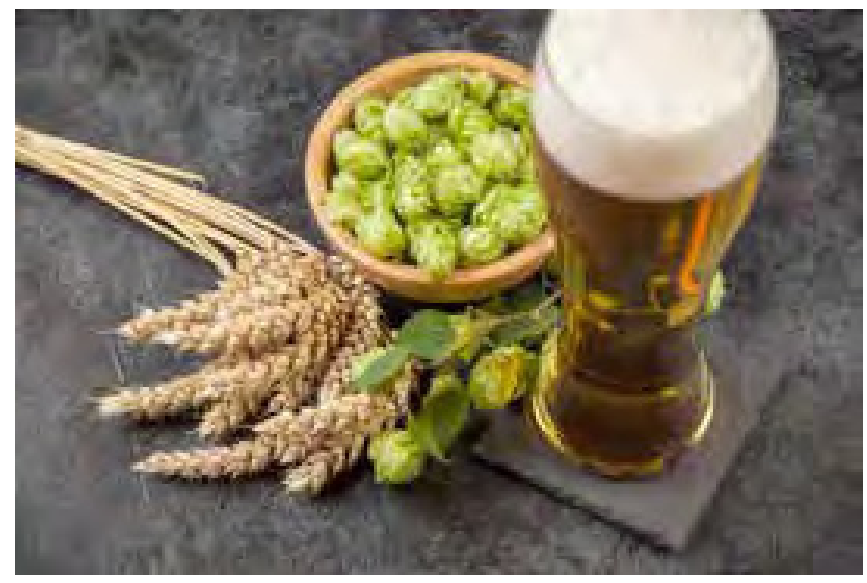

factores y variables a controlar, los métodos utilizados en la producción de cerveza artesanal actualmente en la región no son los más precisos para el control de la calidad del producto, ya que los sistemas implementados en su mayoría son manuales y con grandes rangos de operación dado el bajo dominio de las variables a controlar, las más importantes durante el proceso son los niveles del pH del agua así como el tiempo y temperatura del grano en la maceración ya que éstos ayudan a obtener las enzimas que se requieren dependiendo el tipo de cerveza que se busque obtener.

Las enzimas son proteínas complejas que sirven a manera de catalizadores, induciendo reacciones entre sustancias, estas son activadas o desactivadas bajo ciertas condiciones, de ello surge la importancia de controlar las variables anteriormente mencionadas.

La tabla 1 muestra los rangos óptimos de temperatura que activan las diferentes enzimas del proceso de producción de cerveza [1].

Tabla1. Rangos para activación de enzimas específicas.

\begin{tabular}{|c|c|c|c|}
\hline Enzima & $\begin{array}{c}\text { Rango } \\
\text { Optimo } \\
\text { Temperatura }\end{array}$ & $\begin{array}{c}\text { Rango } \\
\text { Optimo } \\
\text { PH }\end{array}$ & Función \\
\hline Fitasa & $30-52^{\circ} \mathrm{C}$ & $4.4-5.5$ & $\begin{array}{l}\text { Baja el pH del } \\
\text { Mosto. }\end{array}$ \\
\hline $\begin{array}{c}\text { Beta } \\
\text { Glucanasa }\end{array}$ & $36-45^{\circ} \mathrm{C}$ & $4.5-5.0$ & $\begin{array}{l}\text { Reduce la } \\
\text { viscosidad del } \\
\text { mosto, y mejora } \\
\text { la clarificación. }\end{array}$ \\
\hline Peptidasa & $46-57^{\circ} \mathrm{C}$ & $4.6-5.2$ & $\begin{array}{l}\text { Produce Amino } \\
\text { Nitrógeno Libre } \\
\text { (FAN), que es } \\
\text { esencial para } \\
\text { la levadura y la } \\
\text { fermentación. }\end{array}$ \\
\hline Proteasa & $46-57^{\circ} \mathrm{C}$ & $4.6-5.2$ & $\begin{array}{c}\text { Rompe proteínas } \\
\text { grandes y reduce } \\
\text { la turbiedad. }\end{array}$ \\
\hline $\begin{array}{c}\text { Beta } \\
\text { Amilasa }\end{array}$ & $54-65^{\circ} \mathrm{C}$ & $5.0-5.6$ & $\begin{array}{c}\text { Produce azucares } \\
\text { de corta cadena, } \\
\text { altamente } \\
\text { fermentables. }\end{array}$ \\
\hline $\begin{array}{c}\text { Alpha } \\
\text { Amilasa }\end{array}$ & $68-75^{\circ} \mathrm{C}$ & $5.3-5.8$ & $\begin{array}{c}\text { Produce } \\
\text { azucares de larga } \\
\text { cadena, poco } \\
\text { fermentables, } \\
\text { que agregan } \\
\text { cuerpo a la } \\
\text { cerveza. }\end{array}$ \\
\hline
\end{tabular}

Es importante comprender que las enzimas funcionan desde temperaturas por debajo y por arriba del rango 


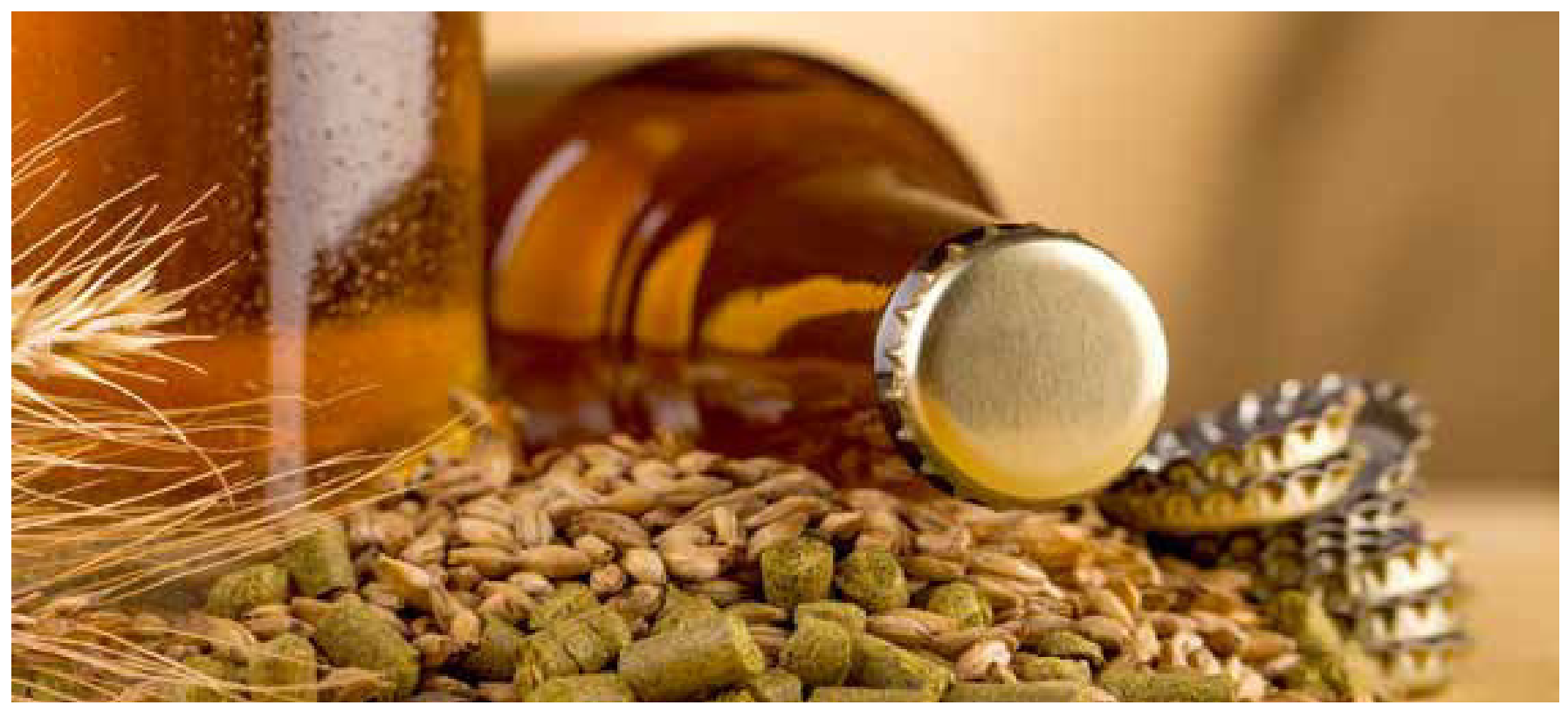

descrito, y que su destrucción toma tiempo, por lo que es posible activar varias enzimas en diferentes puntos de temperatura.

De especial interés es la activación de la Beta-Amilasa para producir azucares fermentables que producen alcohol en el proceso de fermentación, y en la activación de la Alpha-Amilasa para producir azucares de cadena larga (dextrinas), que producen la sensación de cuerpo en la cerveza, por lo cual se optó por macerar a una sola temperatura de $67{ }^{\circ} \mathrm{C}$, manteniendo un $\mathrm{pH}$ entre 5.8 durante 60 minutos, lo que permite obtener tanto azucares fermentables, cómo dextrinas, lo cual se observa en la figura 1 , la cual que muestra la actividad de ambas enzimas y su punto de traslape máximo a $67^{\circ} \mathrm{C}$ [1].

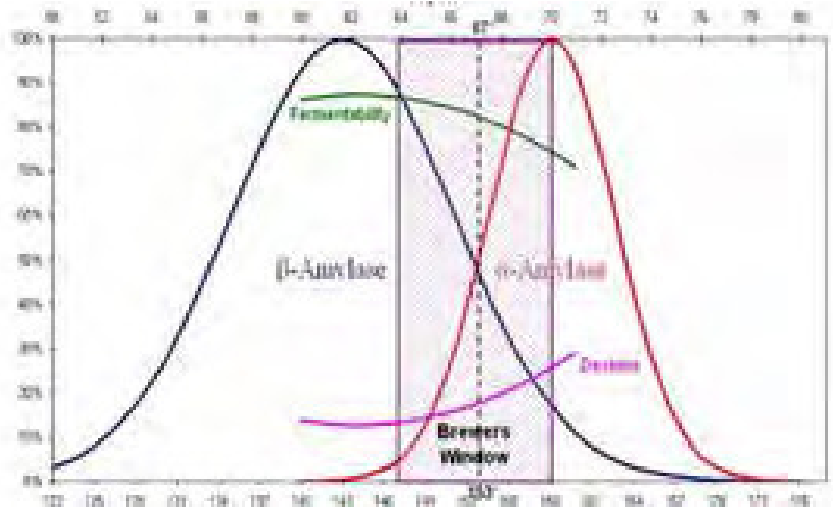

Figura 1. Enzimas activas en una hora bajo las condiciones específicas.

Usar estas condiciones da como resultado cervezas de menor contenido de alcohol, más dulces y de mayor cuerpo.

\section{METODOLOGÍA}

Como se mencionó anteriormente lo que se busca en este proyecto es desarrollar un sistema que controle variables específicas como lo son el valor de $\mathrm{pH}$, los niveles de fluido recipientes y la temperatura, esto permite plantear el problema y a su vez permite visualizar de manera esquemática cada uno de los elementos que conforman el sistema, así como determinar los componentes que se implementaron para cumplir con el objetivo, dadas las especificaciones que debe de tener este sistema

Se tomó la decisión de implementar 3 recipientes cada uno con un propósito específico, el primero al que nombramos recipiente 1 , tiene la función de almacenar el agua ya tratada químicamente con un nivel de $\mathrm{PH}$ especifico actuando a su vez como retroalimentación de la fuente proveedora del líquido donde se estará monitoreando el $\mathrm{pH}$ realizando lecturas con un sensor; si las lecturas arrojan datos dentro del rango deseado se mandara una señal para activar una bomba que moverá el fluido hacia el recipiente 2 , en caso de que no se cumpla la condición y el agua se encuentre fuera de los límites permitidos se mandara una señal a otra bomba que regresara el líquido a la parte inicial del proceso químico, la función del segundo recipiente fue almacenar el agua ya con el $\mathrm{pH}$ requerido a la espera de que se le indique que este fluido pasara al recipiente 3 en donde se realizó el calentamiento quedando el diagrama de siguiente manera:
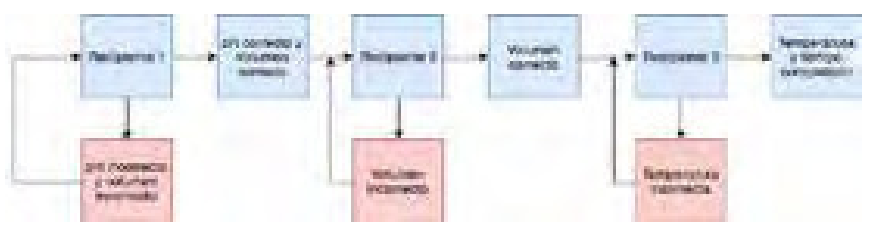

Figura 2. Diagrama del sistema. 


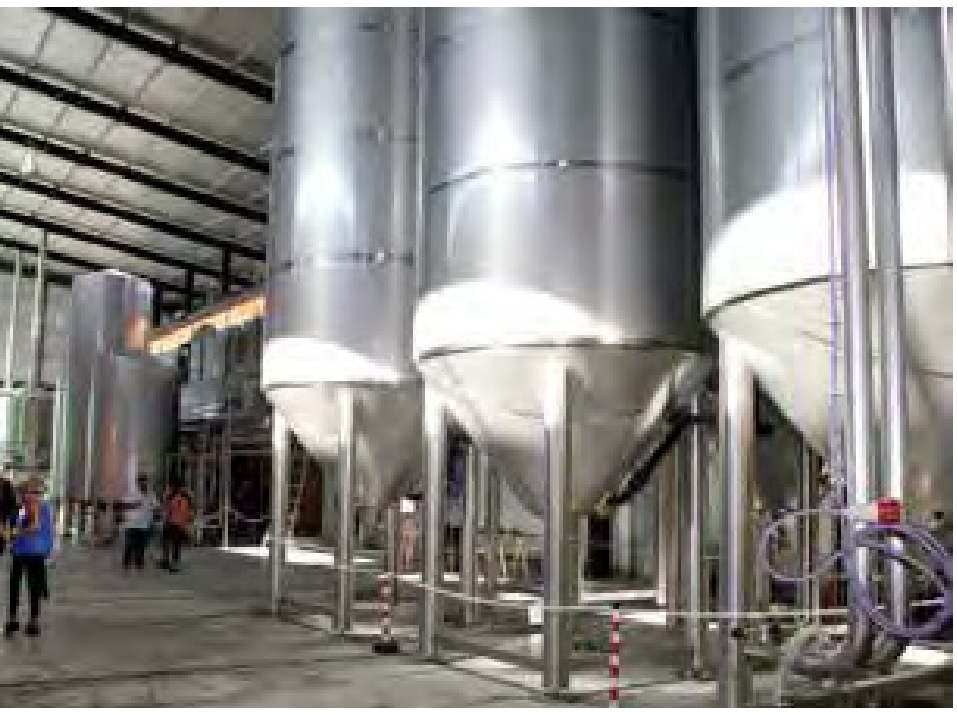

MÉTODO DE TRABAJO

$\mathrm{Al}$ requerirse monitorear 3 variables utilizamos un tipo de sensor específico para cada una de las magnitudes físicas de las cuales queremos tomar lecturas. En el caso del $\mathrm{pH}$ utilizamos $\mathrm{pH}$ Probe de Atlas scientific [6], utilizando su módulo de comunicación I2C para el intercambio de información con la PC. Para el caso del nivel empleamos sensores ultrasónicos modelo HC-SR04, modelo que nos arroja como valor la longitud del sensor al fondo del recipiente, la cual fue multiplicada por el área de la base del recipiente para obtener el volumen. Finalmente para la temperatura utilizamos el sensor analógico Im35 en una versión cubierta de acero inoxidable lo que nos permitió sumergirlo en el fluido y estar en contacto directamente con el producto, estas lecturas se realizaron gracias a la programación utilizando el compilador llamado MikroC en su versión PRO, lo cual nos permitió programar los microcontroladores a los cuales también se les suministro la lógica necesaria para hacer conmutar diferentes salidas bajo especificas condiciones y realizar las tareas otorgadas, los microcontroladores empleados son el PIC 16F84A y el PIC 16F877A y el software para la simulación de los circuitos utilizado fue Proteus 8 profesional, para la etapa de la potencia se utilizaron módulos de relevadores capaces de soportar tenciones de 220v y $10 \mathrm{~A}$ de corriente alterna con una tensión de $5 \mathrm{v}$ de corriente directa para conmutar el solenoide interno, además un circuito para el control de la energía suministrada a una parrilla eléctrica utilizada como fuente de calor con una potencia de 700W; las bombas actuadoras para el traspaso del fluido fueron 2 bombas sumergibles marca jof, modelo BA-180 con una capacidad de 1000 lt/hora.

\section{MONITOREO DEL PH}

El valor del $\mathrm{PH}$ optimo lo definimos anteriormente entre 5 y 5.8 , en este caso el valor que usamos es 5.7 ( $\mathrm{g} g$ " en formato ASCII) debido a que el sensor solo trasmite en ese formato.
El monitoreo lo realizamos con el sensor de $\mathrm{pH}$ de Atlas Scientific el cual trasmitió la información de las lecturas a la PC a través del puerto serial con el protocolo de comunicación I2C, los datos fueron enviados en formato ASCll y recibidos para su lectura mediante una interfaz de usuario codificada en Visual Basic (figura 3), la interfaz fue la encargada de ir recopilando los valores de $\mathrm{pH}$ transmitidos por el sensor y los fue desplegando en la pantalla de la $\mathrm{PC}$, posteriormente los datos son enviados a una base de datos alojados en Microsoft Excel, los valores enviados a Excel se encontraban en formato ASCII y esta base de datos fue programada para que se encargara de hacer la conversión a decimal para poder ser leídos con facilidad, se estableció la programación en VB tal que cuando el sensor le mande el valor de pH óptimo.

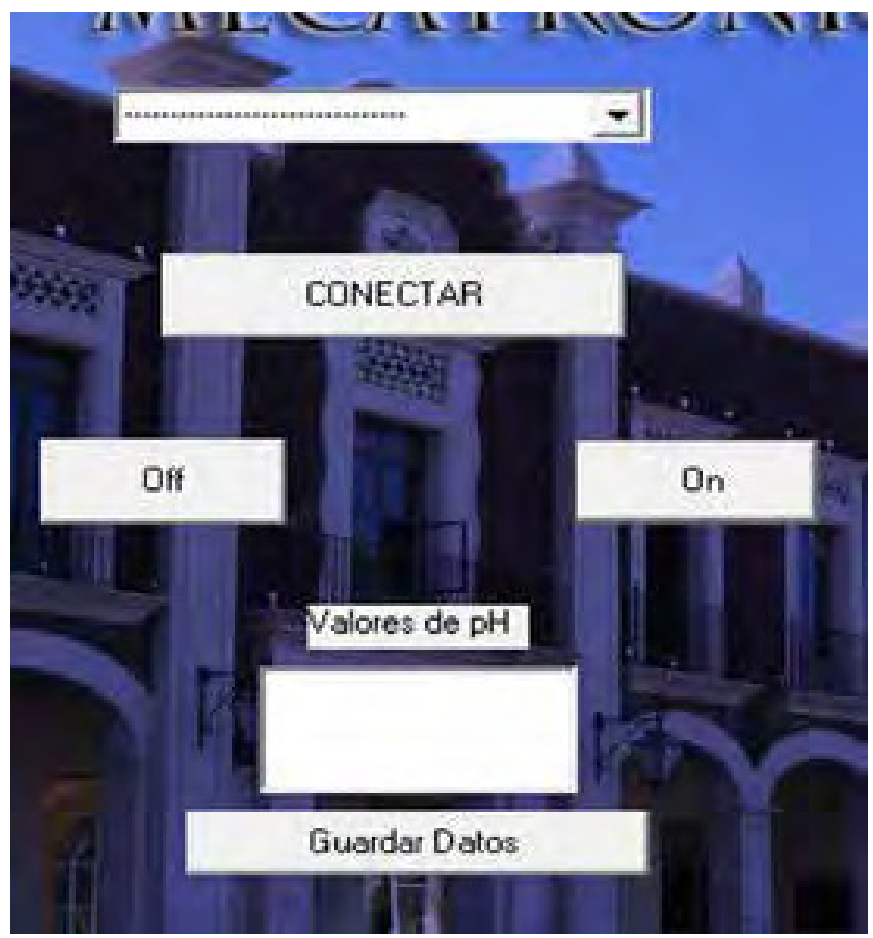

Figura 3. Monitoreo de PH .

En la caja de texto llamada valores de $\mathrm{pH}$ se despliegan los valores enviados por el sensor de $\mathrm{pH}$. Los datos ASCII son separados unitariamente al llegar a la base de datos para posteriormente convertirlos a su valor decimal y poder visualizar el valor correspondiente (Figura 4).

\begin{tabular}{|c|c|c|c|c|c|c|c|c|}
\hline i & 1 & 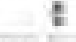 & f & ? & $t$ & 1 & $t$ & $i$ \\
\hline 199 & 24 & $11 \pi$ & 48 & Ises & coss & $73 \pi$ & 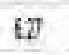 & 速 约 \\
\hline
\end{tabular}

Figura 4. Conversión ASCIl a decimal.

\section{CONTROL DE LOS NIVELES DE LOS TANQUES}

Para esta etapa recurrimos a la implementación de los sensores HC-SR04, estos sensores son capaces de detectar objetos y calcular la distancia a la que se encuentra en un 


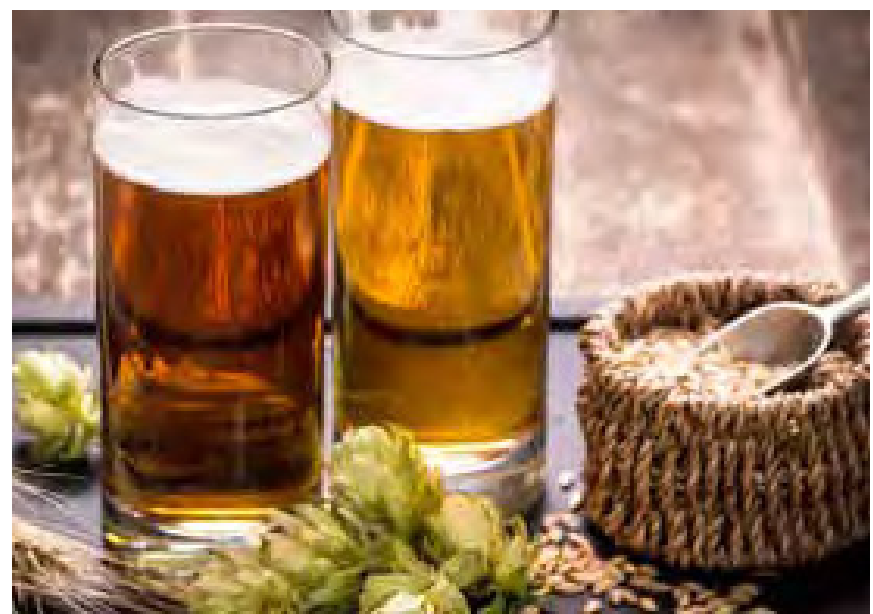

rango de 2 a $450 \mathrm{~cm}$. Este sensor fue elegido por presentar muchas ventajas para el proyecto en particular; es fácil de manipular e instalar por su pequeño factor de forma, su bajo consumo, gran precisión y bajo precio, el sensor funciona por ultrasonido y su uso consiste en enviar el pulso de arranque y medir la anchura del pulso de retorno, las características técnicas que nos ayudaron en la realización del código como en el diseño del circuito físico son las siguientes [2]:

Dimensiones del circuito: $43 \times 20 \times 17 \mathrm{~mm}$

- Tensión de alimentación: 5 Vcc

- Frecuencia de trabajo: $40 \mathrm{KHz}$

" Rango máximo: $4.5 \mathrm{~m}$

" Rango mínimo: $1.7 \mathrm{~cm}$

" Duración mínima del pulso de disparo (nivel TTL): $10 \mu \mathrm{S}$.

" Duración del pulso eco de salida (nivel TTL): $100-25000 \mu \mathrm{S}$.

" Tiempo mínimo de espera entre una medida y el inicio de otra $20 \mathrm{mS}$.

\section{Pines de conexión:}

$\leadsto$ VCC

Trig (Disparo del ultrasonido)

Echo (Recepción del ultrasonido)

$\leadsto$ GND

El control del sensor de ultrasonido se basa en medir el tiempo en el que se activa el pin conectado al Trig del sensor y definir como entrada el pin conectado el puerto Echo y tomar lectura del tiempo de respuesta para determinar la distancia utilizando la siguiente operación

$$
\text { Distancia }=\frac{\left(\frac{\text { Tiempo Trig }}{\text { Tiempo Echo }}\right)(\text { velocidad del sonido })}{2}
$$

Para la implementación de este proyecto utilizamos 2 sensores uno para el recipiente 2 y el otro para el recipiente
3 así como el uso de 2 microcontroladores el 16F84a y el 16F877a respectivamente.

En el caso del recipiente 2 utilizamos un código donde se determina que si la distancia es mayor a $14 \mathrm{~cm}$ o igual se activará un nivel lógico de 1 por un puerto de el microcontrolador condicionado con una compuerta lógica AND con el 1 lógico enviado del PC. Si se encuentra el fluido en un $\mathrm{pH}$ válido, se activa la bomba del recipiente 1 al 2, pero cuando la distancia del líquido es menor o igual a los $15 \mathrm{~cm}$, se apaga el puerto que estaba encendido y activará otro el cual se encuentra condicionado a una compuerta lógica AND junto a una salida del otro microcontrolador que mantiene en alto un nivel lógico si el tanque 3 se encuentra entre el 0 y $100 \%$ de su capacidad. Si estas 2 condiciones se cumplen entonces se encenderá la bomba que llena el recipiente 3 y cuando este llegue al 100\% desactivará uno de los niveles lógicos que van a la AND y desactivaáa la bomba pero, activará otro pin del 16F877a que iniciará el funcionamiento del control de la temperatura, para ilustrar de una manera más grafica el comportamiento deseado, se muestra a continuación los respectivos diagramas de flujo tanto para el sensor ultrasónico del recipiente 2 como el 3:

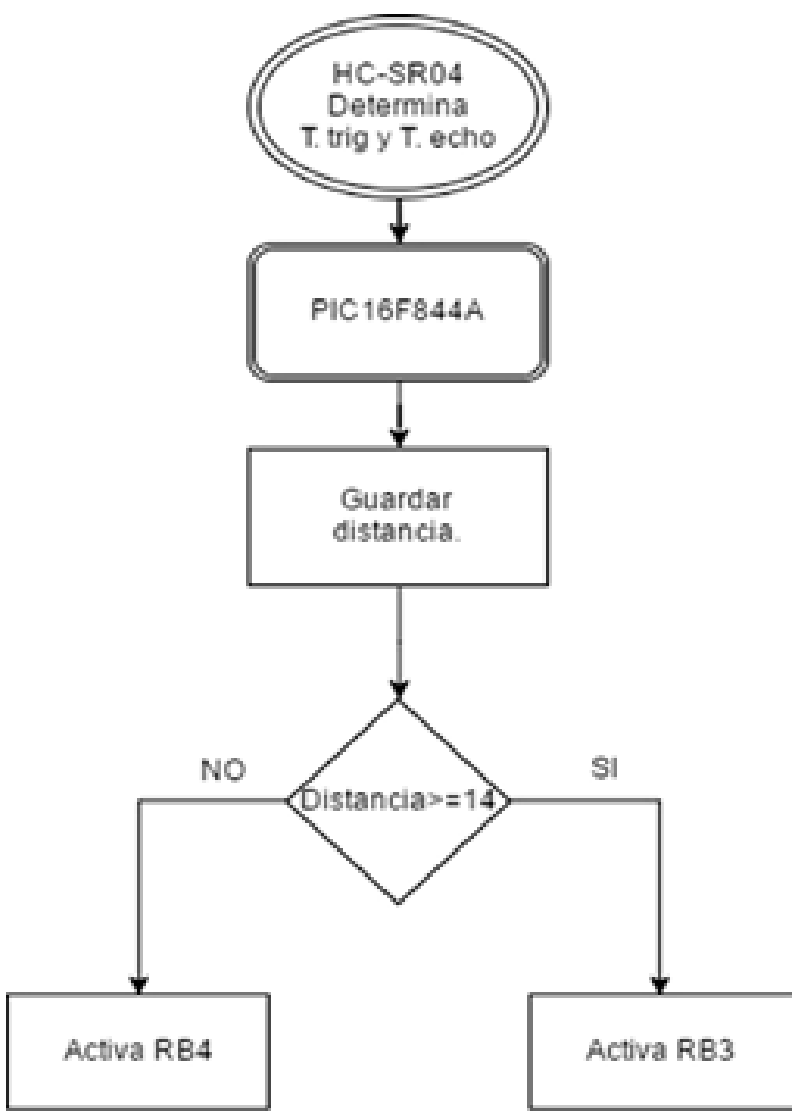

Figura 5. Diagrama de flujo sensor ultrasónico recipiente 2 . 


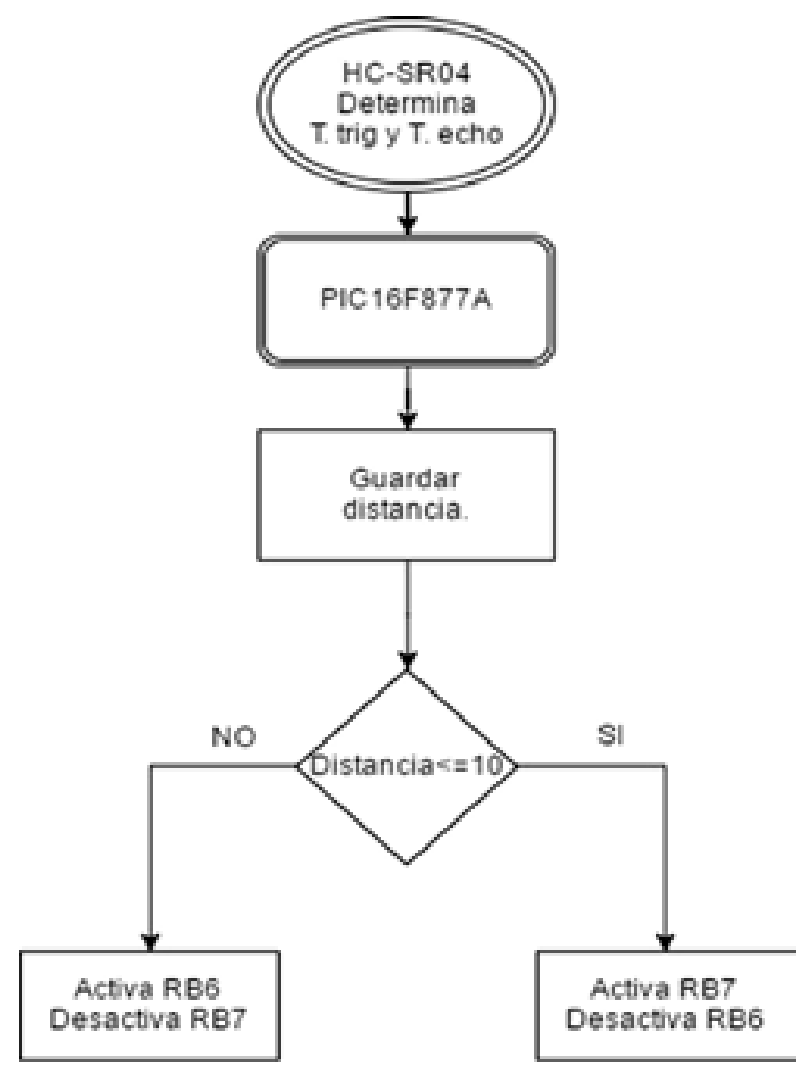

Figura 6. Diagrama de flujo sensor ultrasónico recipiente.

En la siguiente figura podemos observar las conexiones correspondientes y los elementos incorporados al circuito del sensor ultrasónico del recipiente 2

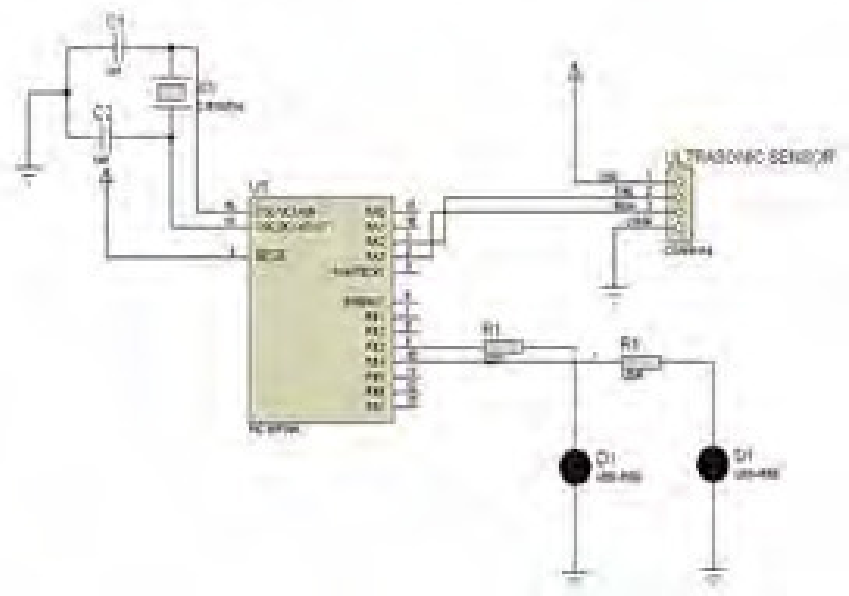

Figura 7. Simulación sensor ultrasónico con el PIC 16 F84a.

El montaje físico de los sensores ultrasónicos requirió que fueran aislados del posible contacto con el agua, realizando la distribución del cableado en el interior de tubos de pvc al igual que el sensor de temperatura, los circuitos fueron montadosy sujetos por medio de soldadura de estaño en placas fenólicas perforadas realizando al cableado y conexiones correspondientes como se mostró en la simulación. Para este circuito configuramos el PIC $16 f 84 a$ con un cristal de cuarzo de $4 \mathrm{MHz}$ y 2 capacitores a tierra y colocamos un LED para cada salida y poder visualizar las conmutaciones de las salidas que van a las compuertas lógicas AND 74LS04 que permite la activación de las bombas una vez cumplidas las condiciones lógicas anteriormente mencionadas

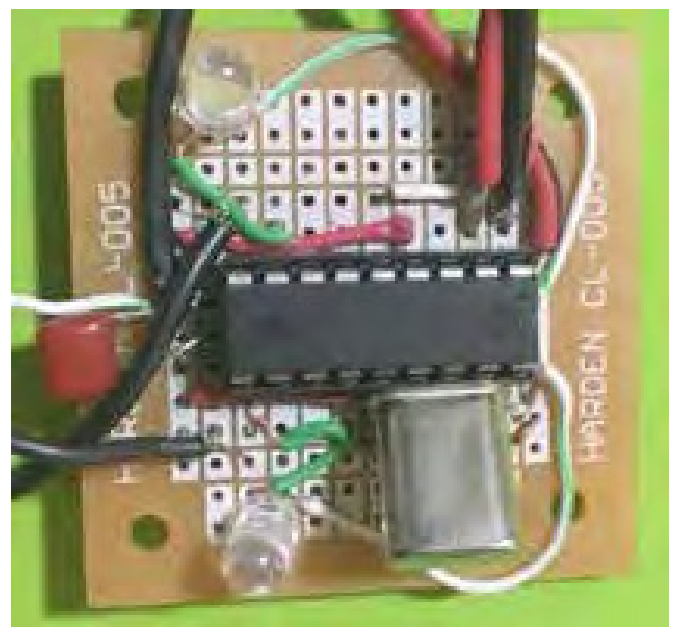

Figura 8. Montaje físico del sensor ultrasónico con el PIC 16F84a.

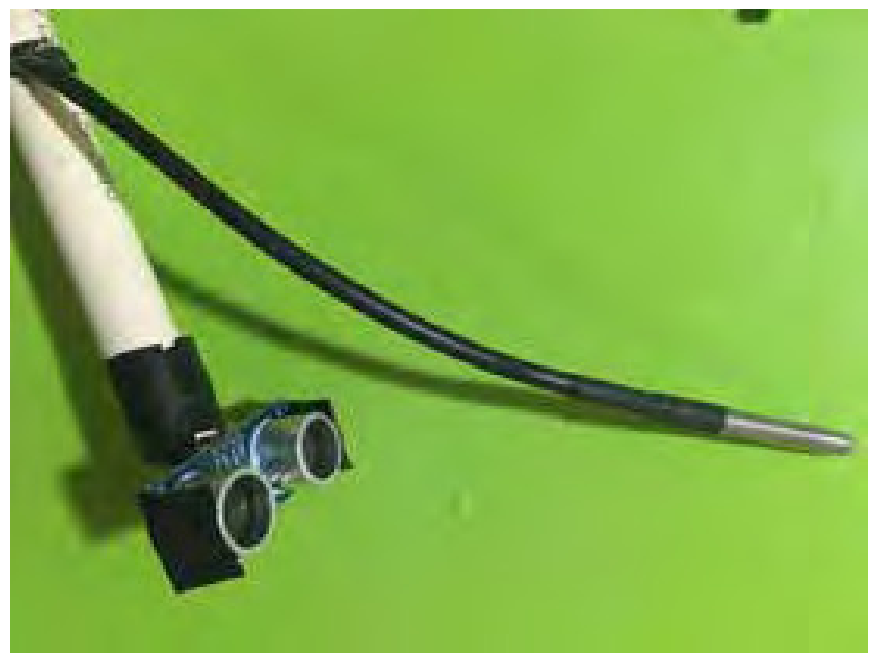

Figura 9. Montaje físico sensor ultrasónico y sensor de temperatura.

A continuación, se muestra la simulación del circuito del sensor ultrasónico para el PIC 16F877a y posteriormente se puede visualizar el montaje físico y operación de el mismo. 


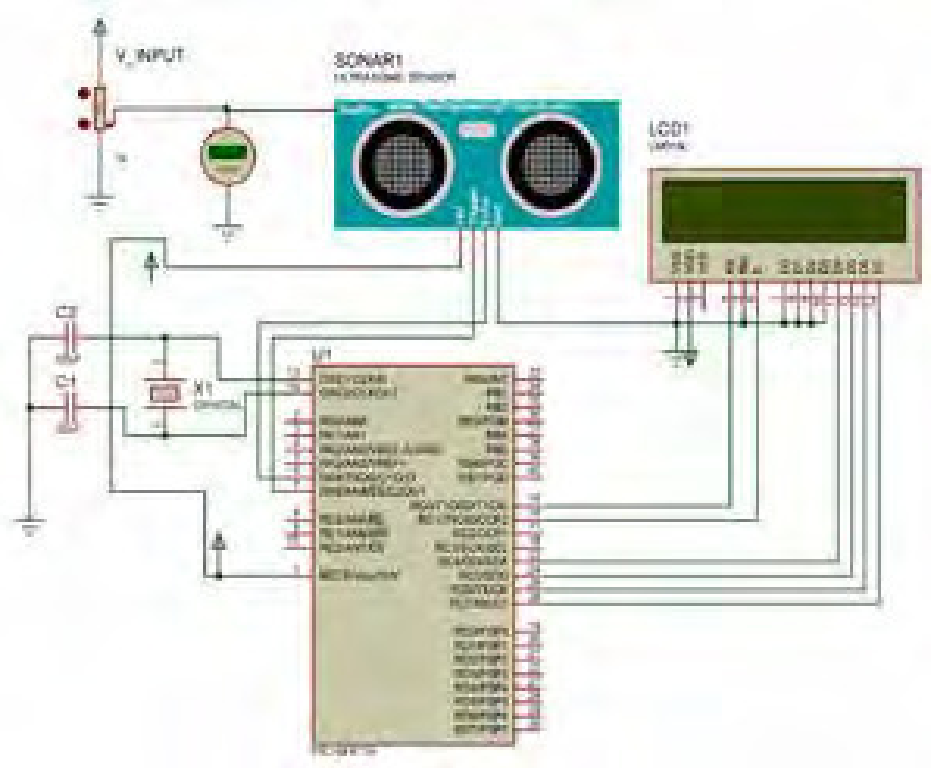

Figura 10. Diagrama de conexión del sensor de ultrasonido.

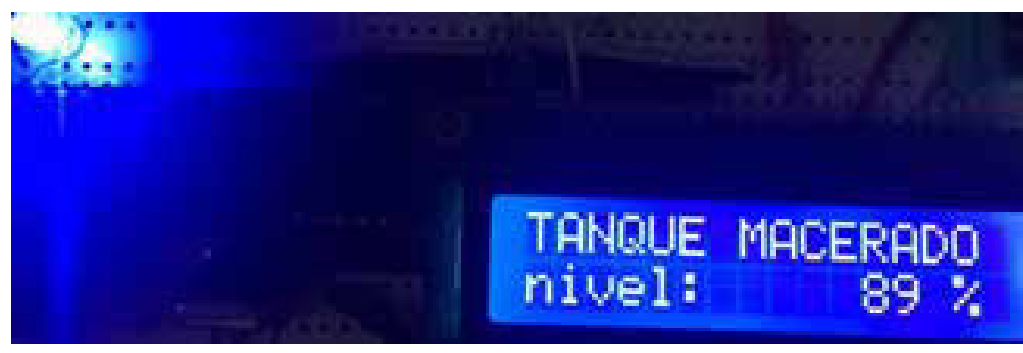

Figura 11. Montaje físico del sensor ultrasónico con el PC 16F877a.

\section{Control de temperatura}

Una vez terminado el llenado de los tanques y dada la condición para el inicio del control de la temperatura, el mismo 1 lógico que inicia el proceso, activa la parrilla eléctrica por medio de otro relevador, iniciando a su vez la señal medida por el sensor $\operatorname{Im} 35$, donde cada grado Celsius equivale a $10 \mathrm{mV}$ [3], por lo tanto puede ser interpretada y obtenida una lectura precisa de la temperatura actual por medio de un pin definido como una entrada analógica en los registros del código para disminuir el error entre el valor deseado y el valor real, este control se logra empleando uno de los puertos configurables como pwm con los que cuenta el PIC 16F877a [4], cuya señal controla el disparo del triac y así controla la potencia consumida por la parrilla y lograr una vez alcanzada la estabilidad del sistema una temperatura estable igual al set point indicado, como se muestra en el siguiente diagrama de bloques:

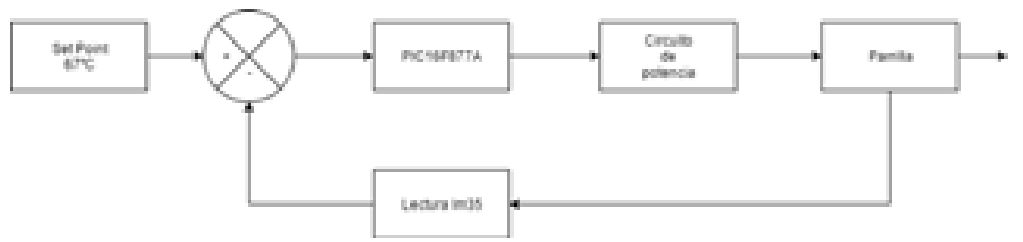

Figura 12. Diagrama de bloques del sistema de control de la temperatura. 
A continuación, se incluye la figura con la simulación del circuito controlador de la temperatura para el recipiente 3 donde se realiza el macerado:

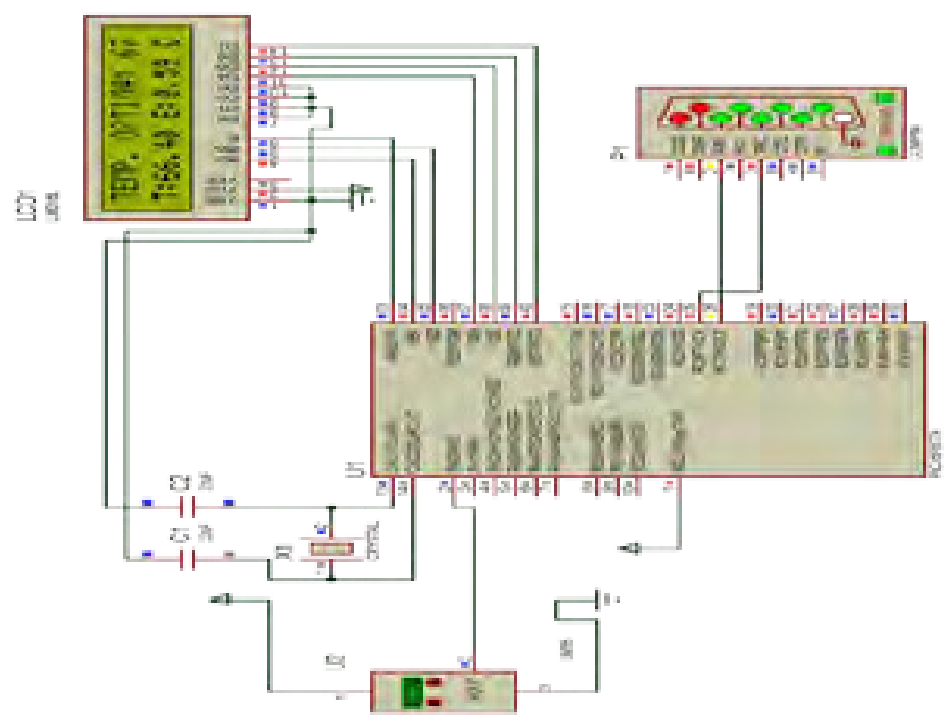

Figura 13. Simulación del control de temperatura con el PIC 16 F877.

En la simulación se puede apreciar las conexiones y componentes que intervienen en el circuito lógico cuya salida del puerto ccp1 se conecta a un opto acoplador como se observa en el circuito de potencia empleado visible en la siguiente imagen [5]:

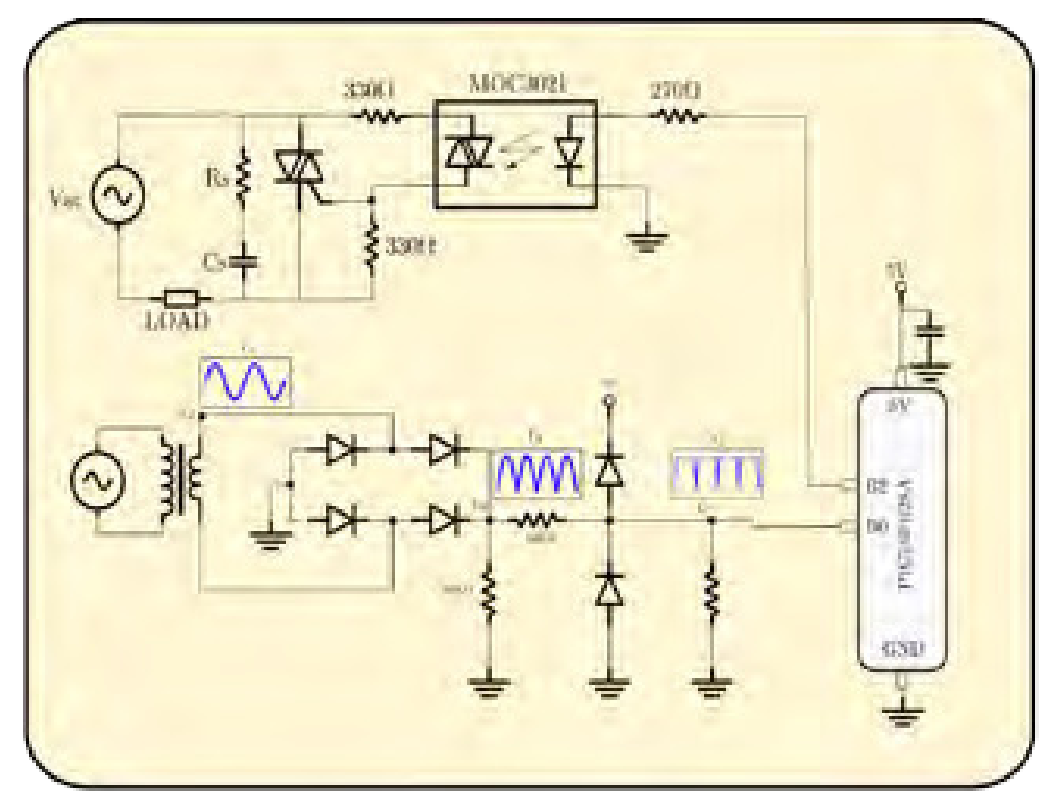

Figura 14. Circuito de potencia para parrilla eléctrica.

A continuación, se muestra los valores reales mostrados en una Icd del montaje físico del circuito 


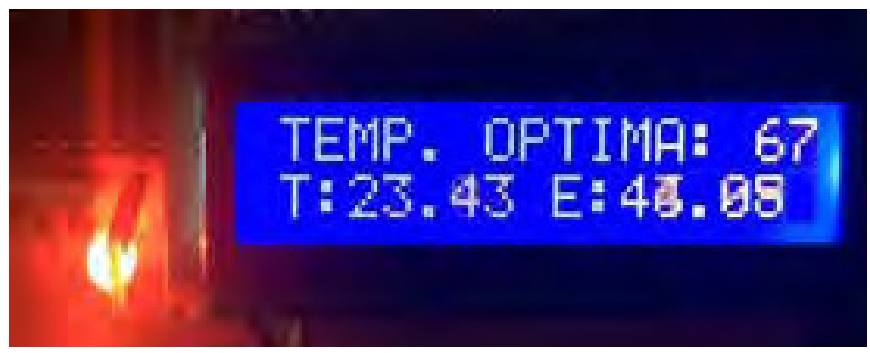

Figura 15. Montaje físico del control de temperatura con el PIC 16F877

Comunicación con la PC e interfaz grafica

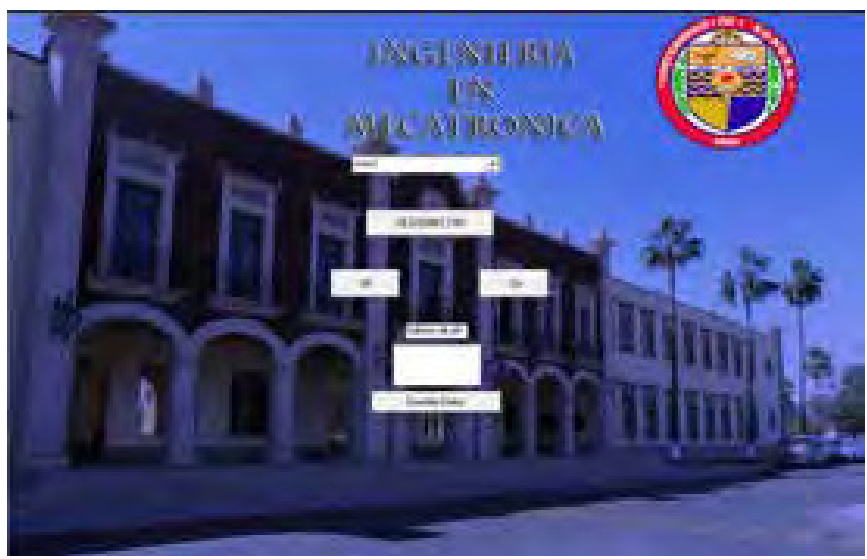

Figura 16. Interfaz grafica

La interfaz diseñada para el proyecto se encarga de tres funciones principales:

Encendido/apagado del sistema

Monitoreo y recopilación de los valores enviados por el sensor de $\mathrm{pH}$.

Control del pH del primer recipiente, si el pH no es el adecuado regresa el contenido a la parte inicial del proyecto, de lo contrario procede a activar una bomba que envía el contenido al recipiente número 2.

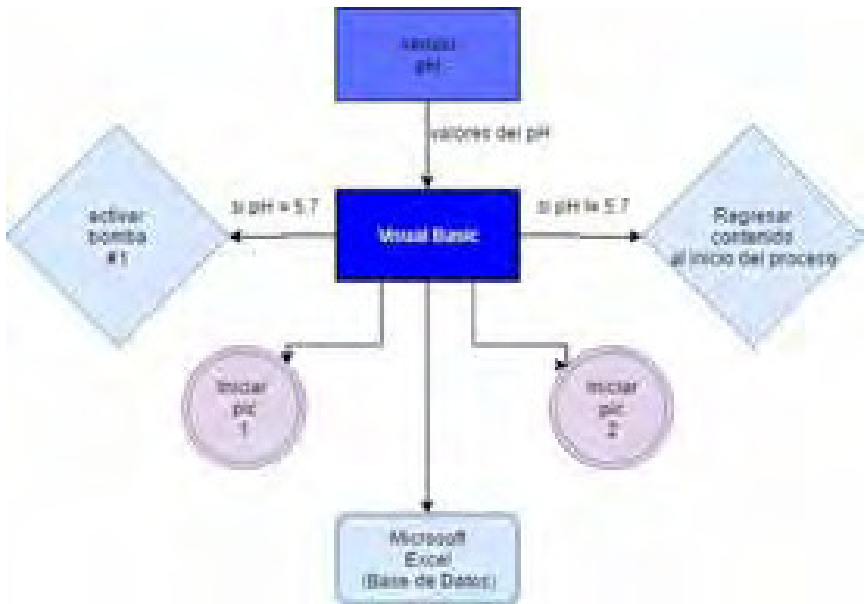

Figura 17. Diagrama de flujo del control por medio del computador.

\section{CONCLUSIONES}

Se logró completar la construcción de un prototipo como se puede apreciar en la figura 18, el cual cumple con las características y funciones para lo cual fue diseñado, dejando abierta la posibilidad de hacer mejoras al sistema como aumentar las instrucciones manipuladas desde la computadora, además anexar más de los procesos necesarios para la producción de cerveza y utilizarse a una producción mayor modificando la etapa de potencia de acuerdo a las necesidades

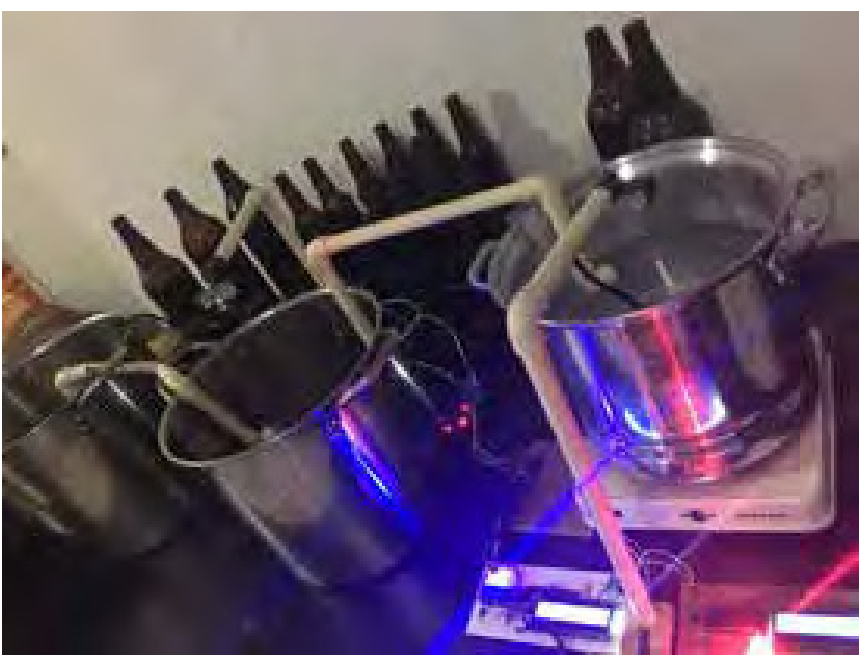

Figura 18. Prototipo puesto en operación.

\section{BIBLIOGRAFÍA}

[1] Juan José García Ornelas, 'Las Temperaturas de Maceración, Ciencia y Arte". En línea, publicado el 2 de julio de 2013 en: http://brewmasters.com.mx/las-temperaturas-demaceracion-ciencia-y-arte/

[2] Kevin Soria, "HC-SR04 Sensor Ultrasónico". En línea, publicado el 17 de septiembre del 2013 en: http://bkargado. blogspot.mx/2013/09/todosobrehc-sr04.html

[3] Raúl González, "El Sensor de Temperatura LM35".En línea, Publicado el 26 de octubre del 2009 en: http://pcexpertos. com/2009/10/el-sensor-de-temperatura-Im35.html

[4] Sergio Castaño, "17. Control PID con micro controlador PIC". En línea, publicado el 15 de febrero del 2016 en: http:// controlautomaticoeducacion.com/17-control-pid-conmicrocontrolador-pic/

[5] Jonny Cristhian Otero Baca, "Control de Potencia en AC con triac y micro controlador PIC 16F628A".En línea, publicado el 3 de febrero del 2014 en: http://voltiosybytes.blogspot. $\mathrm{mx} / 2014 / 02 /$ control-de-potencia-en-ac-con-triac-y.html

[6] https://www.atlas-scientific.com/product_pages/probes/ ph_probe.html, publicado el 20/07/2017

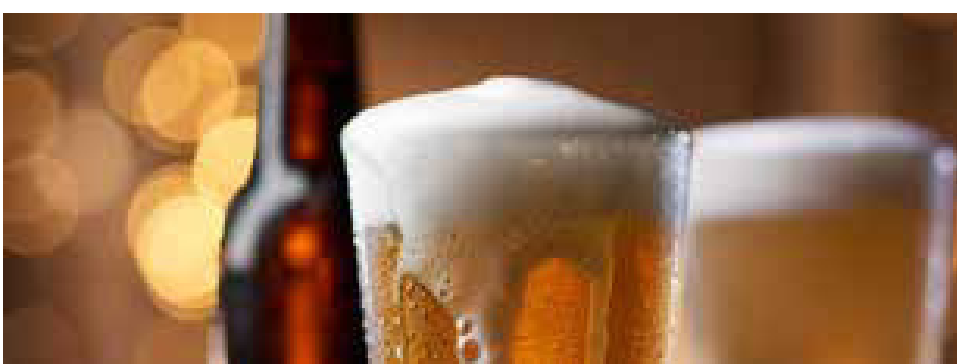

